Physical Sciences | The COOLscience Club

\section{Exploring the origins of life with autocatalytic sets}

How did we get here? This question has plagued philosophers, scientists and individuals alike for hundreds, if not thousands of years.

humans, are incredibly complex systems, even down to the microscopic cellular tevel, and understanding our own origins of life and how our DNA came to be what we know today is a challenging question. The $\mathrm{COOLscien}$ Club is an international collaboration investigating how self-replicating systems called autocatalytic sets. a trillionth of a second, still has many however, are not so well established. ne of the most popular
theories about the beginnings
of our universe is the Big
Bang theory. This is the idea that the universe started as an infinitely violently expanded within just a few fractions of a second. While the exa beginning of the universe, what happened within the first trillionth of mysteries, scientists now have many compelling models and evidence for what happened in the instants just after our universe was born.

The beginnings of life as we know it, Evolved life represents an incredibly complex mixture of chemistry and biology, with the whole human 204 billion atoms. For comparison, a water molecule contains just three. re still those in the fullerene family which are strange, football-shaped the largest of these contains just 70 atoms.

Fullerenes aren't found everywhere. Many planets only have very simple molecules, composed of less than 10 atoms. So how did such simple chemical building blocks come together to create the complexity and variety of chemical species we have on Earth, including the chemical compounds like DNA that are responsible for life?

Professor Stuart Kauffman, a retired bu still highly active biologist, is interested have be macly this question. There on how complex life cang to be but he is interested in the ro be, autocatalytic sets may have played The largest molecules found in space in the formation of complex chemical

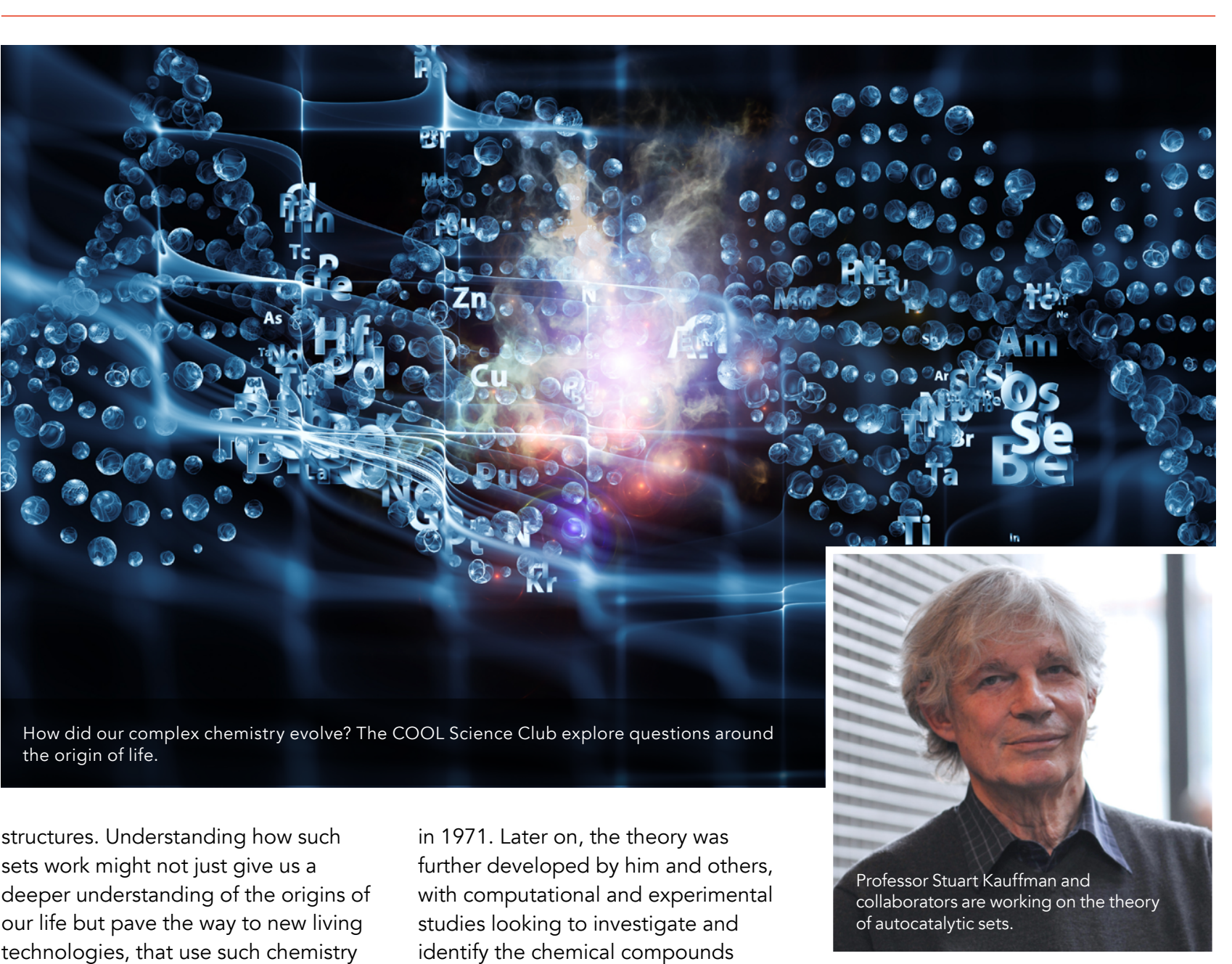
as part of their function and growth.

SELF-SUSTAINING CHEMISTRY The concept of an autocatalytic set is a set of chemical reactions that form self-sustaining network. As long as identify the chemical compounds necessary to form autocatalytic sets or small organic molecules.

Now Professor Kauffman is working with a number of collaborators to take an interdisciplinary approach

Life is more than the sum of its constituent molecules. It depends on how these molecules interact with each other.

within such a reaction network are to the problem of autocatalytic capable of mutually catalysing each sets. By combining methods from other's formation. This means that individual molecules do not need to be capable of catalysing their own formation as this can be done by another molecule in the network. The end result is a combined set of reactions that are fully self-sustaining. Kauffman originally proposed the concept of autocatalytic sets and their
potential role in complex evolution sets. By combining methods from biology and computer science, the group aim to better understand the conditions necessary for an autocatalytic set to form and then how such sets can evolve and grow. Such an explanation might not just hold the answer for how primordial soup led to RNA, DNA and complex cellular sustaining technologies.
RNA - NATURE'S DECODER Originally, the concept of autocatalytic sets was a controversial one. One of the dominant views in origin-of-life research is the RNA world hypothesis. RNA is another type of nucleic acid like DNA but, instead of storing all genetic mformation as DNA does, RNA acts information contained in the DNA and convert it into actionable instructions for cells.

In the RNA world hypothesis, RNA alone was initially responsible for genetic information, meaning it would have to be capable of self-replication. Only recently has evidence of selfreplication in short RNA strands been found, but there is no evidence (yet) that RNA can catalyse its own templatedirected replication so RNA could be replicated and express genetic

If autocatalytic sets are instea considered to be responsible for life, both the replication and expression of 


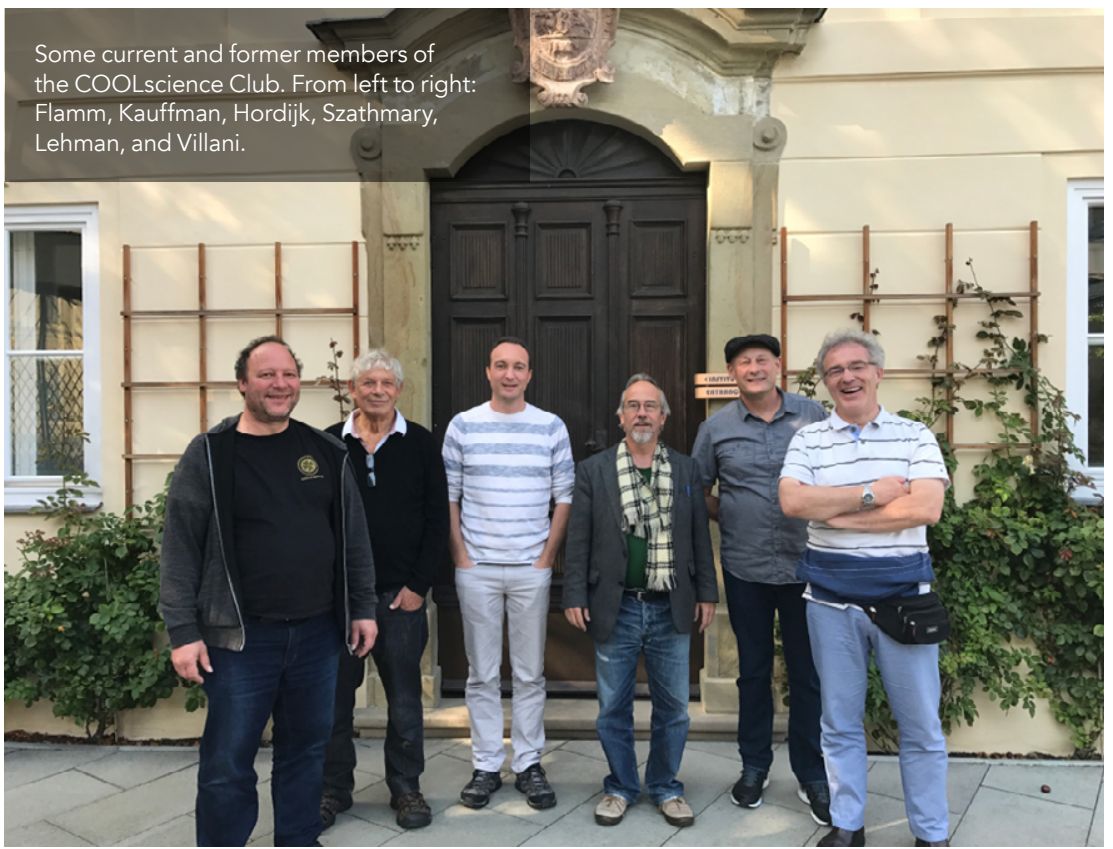

The origin of life is a complex problem, requiring a highly interdisciplinary approach.

RNA does not need to be capable of both regulation and self-expression. However, some critics of the hypothesis have argued that it is difficult to see how highly complex networks of reactions could arise themselves spontaneously, or how the set could evolve and adapt without dissupting the fine balance of reactions needed for the set as a whole to function. Many agree, though, that even if such sets aren't responsible for at of the origin of ile, they are a plausib a

One of the key developments in helping to establish the potential and power of autocatalytic sets was the work of Prof Mike Steel and Dr Wim Hordijk on a formal mathematical framework to describe autocatalytic sets, known as Reflexively Autocatalytic and Foodgenerated (RAF) theory. This helped to capture in a more mathematically form way the potential ways autocatalytic sets could form by explicitly defining which molecules catalyse the formation

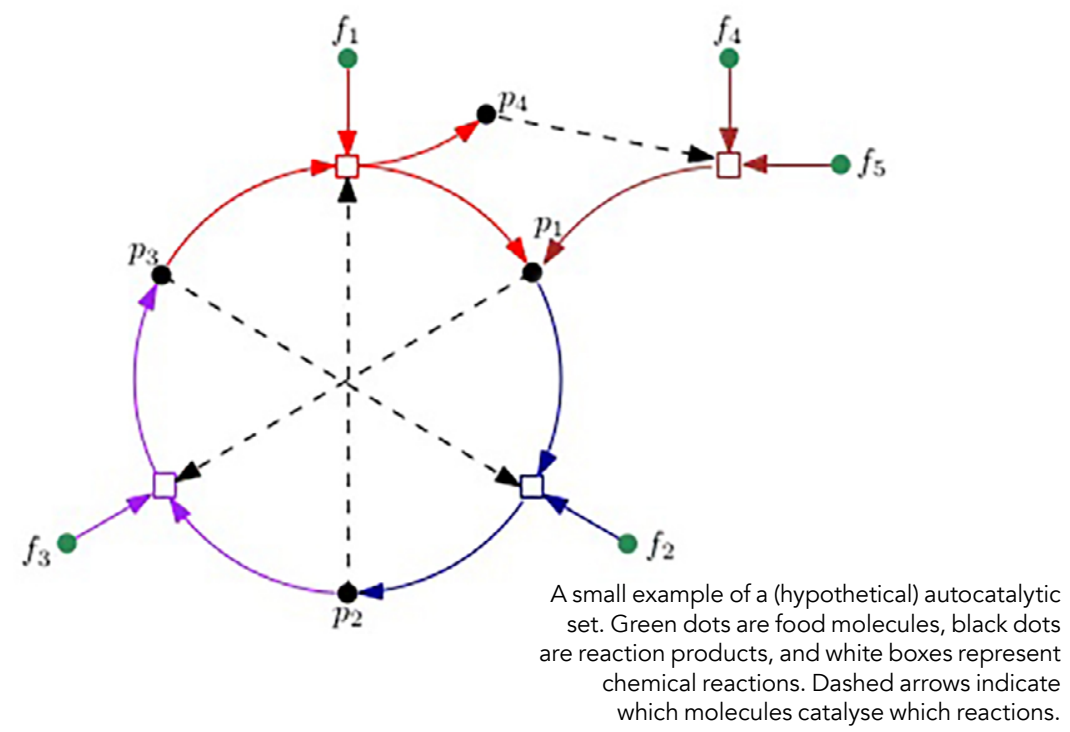

f which other molecules - and a 'foodsubset - which molecules an be created from the food source.

This mathematical description paved the way for more detailed theoretical and computational work and revealed two key things: how these sets could form spontaneously from a given food source, and how they could potentially evolve and diversify. These sets are not just restricted to the reactions involved in the origins of life either Groups have looked at the set of proctions involved in the metabolic prosses of particular archaeagenerated sets have been successfully constructed since the mid-90s.

\section{SUM OF PARTS}

An autocatalytic set is something where the system is truly greater than the sum of its parts. Even if you put all the celluta components of bacteria in a solution they will never spontaneously assemble to have the capabilities of a bacterial. cell. However, when all the components work together as part of this complex network, a series of genetic information cell walls and so on suddenly become something highly sophisticated, complex and living.

While the individual reactions in set may not be particularly useful in isolation or make the desired fina products of a reaction, combining is key to uncking their potential o synthesise complex life. As the underlying theory of autocatalytic sets laid out by Prof Kauffman and colleagues is fully extendable to larger networks, and is highly flexible and can be applied to any related problems, then it is possible to think of using this methodology to deliberately target the ceation of certain molecular species.

As part of an EU joint project, Prof Kauffman and colleagues are hoping to harness this theory, in collaboration with industrial partners, to identify potential reactions, sequence molecules and detect products. This research could of used to not just identify the origins for new types of automnod synthesis.

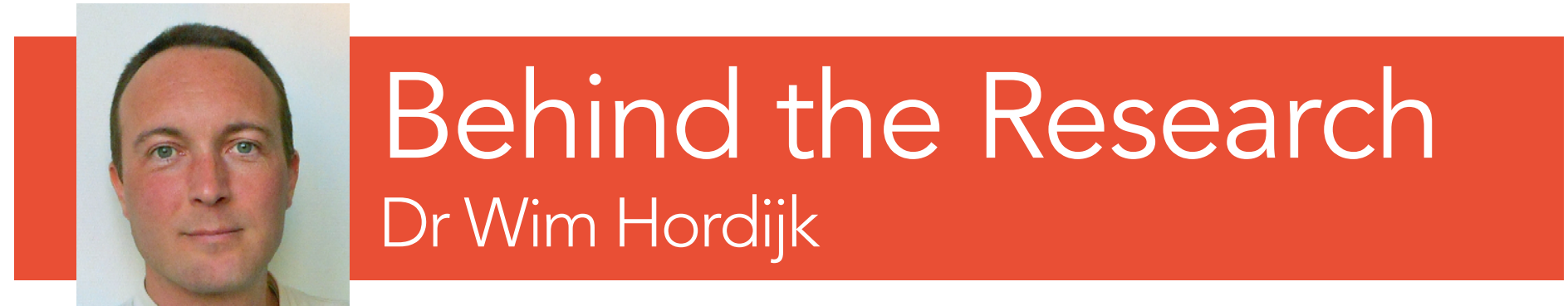

E: wim@WorldWideWanderings.net W: WorldWideWanderings.net

\section{Research Objectives}

The Cooperative Origin Of Life ("COOL") science club investigates the origin of life, particularly with

\section{Detail}

Bio

The COOLscience Club is an international and interdisciplinary collaboration of scientists studying the origin and early evolution of life in terms of chemical reaction networks involving several different types of molecules that cooperatively and collectively are self-reproducing, rather than focusing on the properties and synthesis of single molecule types.

\section{Funding
ATTRACT}

https://attract-eu.com/

Collaborators

COOLscience Club

https://coolscience.club/

$$
\text { (6) CoOLscience Club }
$$

\section{References}

Hordijk, W. (2019). A History of Autocatalytic Sets. Biological Theory. https://doi.org/10.1007/s13752-019-00330-w

Hordijk, W., \& Steel, M. (2017). Chasing the tail: The . Biosystems, 152, 1-10. https://doi.org/10.1016/j.biosystems.2016.12.002

Hordijk, W. (2019). How did life begin? Orbiter. https:// orbitermag com/how-did-life-begin-part-1/

\section{Personal Response}

What areas of autocatalytic sets will you be

focusing on in your future work?

TT have autocatalytic sets emerge and evolve spontaneously in a controlled laboratory setting. This could be a prebiotically relevant setting to study the spontaneous synthesis of chemically or biologically
"useful" products and reaction networks.

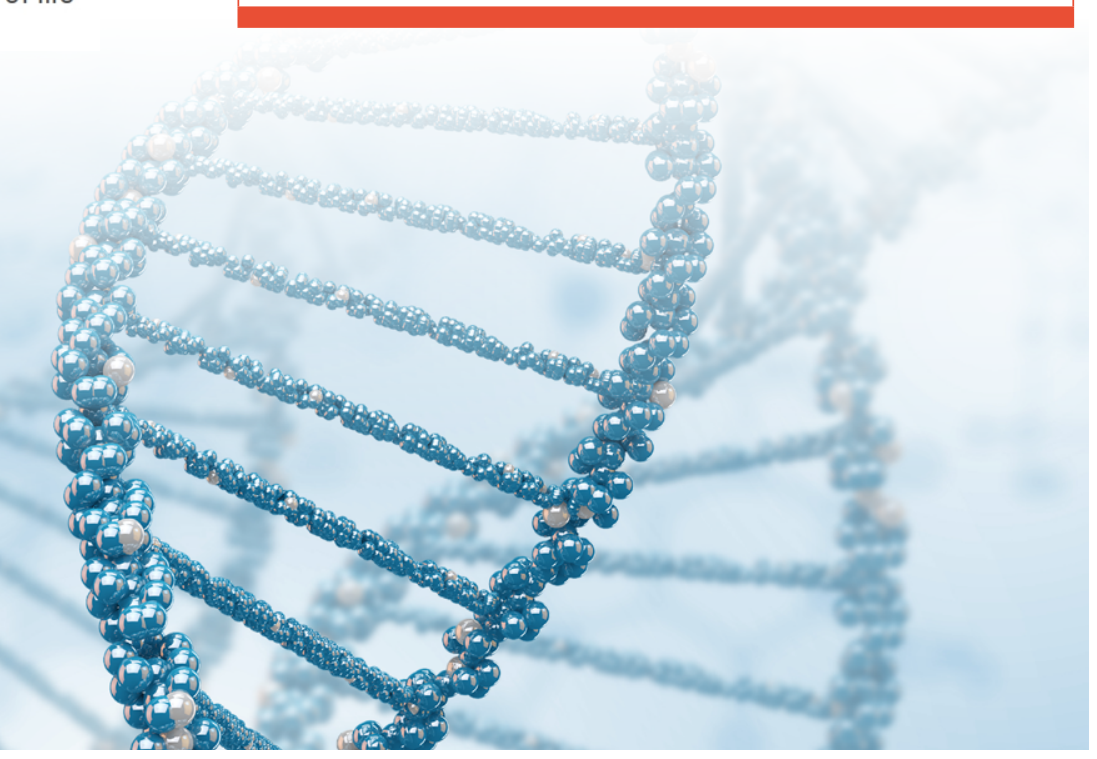

